\title{
Research on A Multi-needle Grounding Pole
}

\author{
Zhang Lei ${ }^{1}$, Liu Guobin ${ }^{1}$, Zhang Jiayan ${ }^{1}$, Liu Xiao ${ }^{1}$, Yang Xinkun ${ }^{1}$, Wang Miao ${ }^{1}$, Li \\ $\mathrm{Na}^{1}$, Shi Feng ${ }^{1}$, Zhang $\mathrm{Bo}^{1}$, Li Tianci ${ }^{1}$, Huang Dongwei ${ }^{1}$, Wang $\mathrm{Yi}^{1}$, Liu Zhitong ${ }^{2}$ \\ ${ }^{1}$ Fushun Power Supply Company, Liaoning Electric Power Company Limited, State Grid, China, \\ ${ }^{2}$ Shenyang Power Supply Company, Liaoning Electric Power Company Limited, State Grid, China, \\ fushunpowersupply@163.com
}

Keywords: grounding resistance; current voltmeter method; grounding resistance meter method; the multi-needle grounding pole; application on spot

\begin{abstract}
Grounding technology plays an important role in ensuring safe and reliable operation of the power system and the personal safety. Grounding resistance as an important ground parameter, dictate the degree of electrical safety. This paper introduces the basic principles of testing the grounding resistance, the testing methods and the necessity. According to the actual application and comprised data. This paper introduces the advantages of the multi-needle grounding pole, and it can be applied to the rock, the frozen ground and the other hard permafrost soil. Using it, we can make the ground resistance testing would not be affected by the seasonal environment influence, and it can be applied to the grounding resistance test in the electric power and mining enterprises.
\end{abstract}

\section{Introduction}

In recent years, many substations in Fushun have expanding accidents caused by the lightning .Most of the accidents is associated with the unqualified grounding resistance. Grounding net plays an important role on working grounding and protective grounding. When the grounding resistance is too large, it will cause an accident. It will increase the neutral point voltage offset, and it may cause sound phase and the neutral point voltage over high, and it will exceed the level of the insulation requirements and cause equipment damage[1-3]. When lightning or thunder waves attacks, Current increases, which will produced a high residual voltage. The equipment nearby will be threatened by the counterattack voltage. Apart from this, the quality of grounding resistance is directly related to the safety of operation and maintenance staff. Therefore, we must strengthen the regular monitoring of grounding resistance, thereby ensuring safe and reliable operation of the power grid[4, 5].

\section{The basic principle of grounding resistance test}

Grounding resistance refers to it when the current through the grounding body flow into the soil, that will form the resistance by the soil around the grounding body. Grounding resistance add the resistance of attachment between grounding body, grounding body itself , the resistance between grounding body and soil .The numerical value is the ratio of grounding body voltage to the earth and the current through the grounding body. If the resistance is calculated with operation frequency alternating currents, we call it operation frequency grounding resistance. If the resistance is calculated with the surge currents through the grounding body flow into the earth, we call it surge grounding resistance.

\section{The basic method of grounding resistance test}

(1)Current voltmeter method

Current voltmeter method applies to measure large power station or transformer substation grounding grid grounding resistance. By the current voltmeter method, the test wiring of measure grounding resistance is shown in figure 1. 


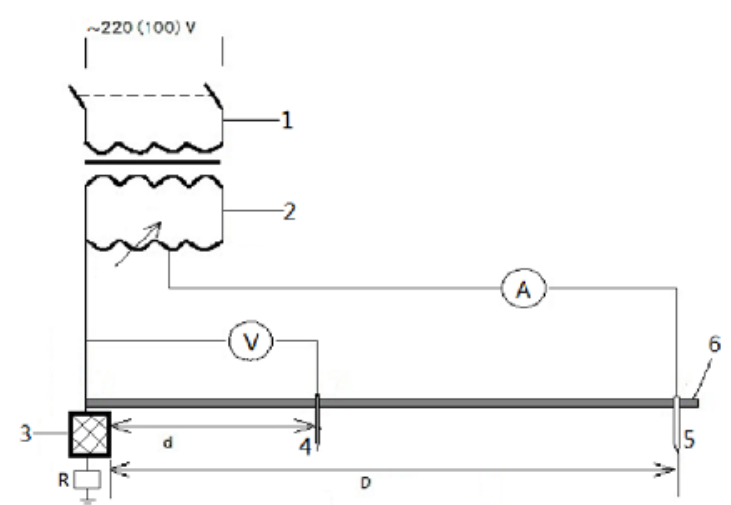

Fig. 1 the test wiring by the current voltmeter method

1-isolation transformer $\quad 2$-autoformer regulator 3 -grounding grid

4-Auxiliary voltage pole 5-Auxiliary current pole 6-earth surface

Usually, according to the pole arrangement when we test the grounding resistance, we take the 0.618 and $30^{\circ}$ testing method. Voltage current line arrangement (0.618 testing method), Generally we select current line $\mathrm{D}$ for the largest diagonal in grounding grid length of $4 \sim 5$ times, voltage line $\mathrm{D}$ is about $0.618 * \mathrm{D}$. Voltage current triangle arrangement $\left(30^{\circ}\right.$ testing method), To compare with line arrangement, Voltage current triangle arrangement has the advantages of little influence on mutual inductance between down-lead. At this point, we general take $\mathrm{d}=\mathrm{D} \geqslant 2$ times the length of the diagonal, $\theta \approx 30^{\circ}$.

(2)Grounding resistance meter method

Grounding resistance meter method generally apply to transmission line tower, independent lightning rod, microwave tower and other small grounding device ,grounding resistance measurement.

Normally we adopt ZC-8 grounding resistance meter to measure grounding resistance, the connection is shown in figure 2.

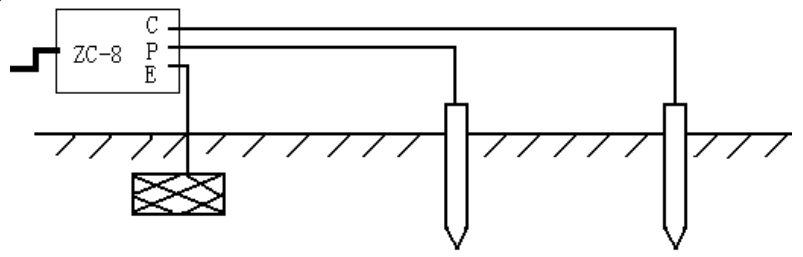

Fig.2 ZC-8 grounding resistance meter measuring grounding resistance

\section{The necessity of grounding resistance testing}

Check whether the new input grounding grid accord with the design rules. During operation ground lead and null line sometimes were affected by the damage of external force or chemical corrosion, etc. Damage or fracture phenomenon is always happen. The soil surrounding the grounding body will also be changed, because the drought and freezing affect the grounding resistance. In order to ensure the reliability of ground connection and connect to neutral, we must make regular inspection and test on the grounding device.

\section{Problems on site testing}

At present, we use one or more steel tube whose diameter is $25-50 \mathrm{~mm}$, length is $500 \mathrm{~mm}$ for voltage (current) grounding in $10 \mathrm{kV}$ system grounding resistance testing. We insert it vertically into the ground about $400 \mathrm{~mm}$. Fushun is located in the mountains, the soil is very hard, especially in the winter the soil will form the permafrost. Grounding pole is hard to insert into soil, after inserting reluctantly, grounding pole cannot contact with the soil tightly, so that we cannot get the real data of grounding resistance. It directly affects the engineering construction progress, delivery of current and recovery operation, etc. 


\section{The study on grounding pole design and data analysis}

According to the above problem, this paper introduced two kinds of grounding pole(Inflatable type and pin type),we use the power plant (electric pick)insert the grounding pole into the soil and start the expander (or pin).

\section{The inflatable type grounding pole}

It use the swivel nut to drive the spring, and make the inflation portion of grounding pole come out and contact with the soil closely. After the test, we tighten the expander, in order to get the grounding pole. The mechanical design and real products picture of the inflatable type grounding pole are shown in Fig.3 and Fig.4.
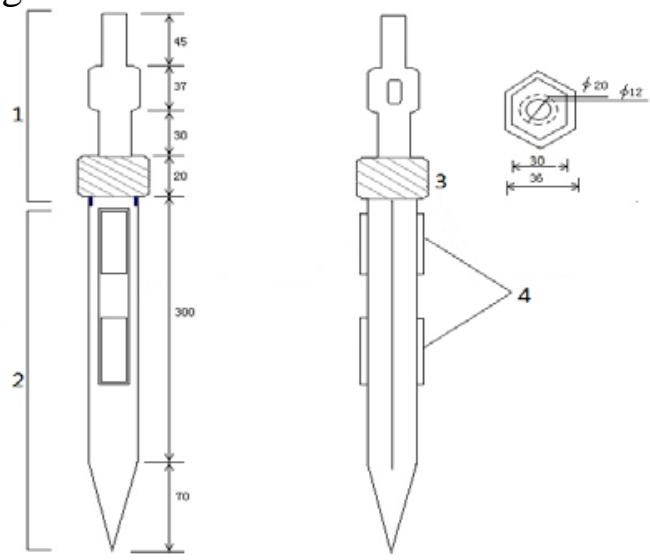

Fig.3 the mechanical design of the inflatable type grounding pole

Note: 1-- Connection to the power plant, 2-- the portion inserted into soil

3-- connecting nut, $\quad 4$-- the portion of inflation and shrinkage

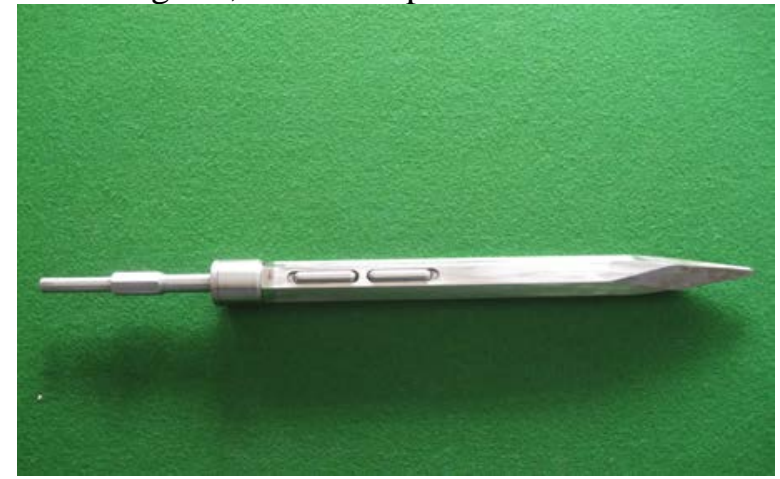

Fig.4 Picture of the inflatable type grounding pole

We choose A, B, C three testing spots in Fushun. We respectively using regular grounding pole and the inflatable type grounding pole on test of grounding resistance, the measured data in the following table.

Table 1 The comparison table of grounding resistance test data

\begin{tabular}{|c|c|c|c|c|}
\hline Item & $\begin{array}{c}\text { Regular grounding } \\
\text { pole }\end{array}$ & \multirow{2}{*}{$\begin{array}{c}\text { Testing } \\
\text { time }\end{array}$} & $\begin{array}{c}\text { the inflatable type } \\
\text { grounding pole }\end{array}$ & \multirow{2}{*}{$\begin{array}{c}\text { Testing } \\
\text { time }\end{array}$} \\
\hline A zone & $6.7 \Omega$ & \multirow{3}{*}{$7 \mathrm{~min}$} & $7.1 \Omega$ & \multirow{2}{*}{$3 \mathrm{~min}$} \\
\cline { 1 - 1 } B zone & $5.8 \Omega$ & $6.2 \Omega$ & \\
\hline C zone & $6.2 \Omega$ & & $6.5 \Omega$ & \\
\hline
\end{tabular}

From the data in the table, in the cold winter, the silo is very hard. When we use the regular grounding pole to test, it is very difficult to insert it, and it took a long time foe testing. When we use the inflatable grounding pole to test, due to the application of the electric device, grounding pole insult the soil smoothly, and testing time is significantly shortened, but the grounding resistance value is increases.

According to the analysis results, we research from many factors, finally we focus on the expansion portion of the inflatable type grounding pole. In the process during the inflatable type 
grounding pole insert the soil, because of the electric device, grounding pole had a strong vibration. The side of grounding pole cannot contact with the soil closely. So the test result is not accurate, failed to achieve the desired test results.

According to the shortcoming of the inflatable type grounding pole, we redesigned and developed the multi-needle grounding pole.

\section{The multi-needle grounding pole}

It use the swivel nut to drive the spring, and make the steel needle of grounding pole insult into the soil. After the test, we shrink four steel and remove grounding pole. The mechanical design and real products picture of the multi-needle grounding pole are shown in Fig.5 and Fig.6.
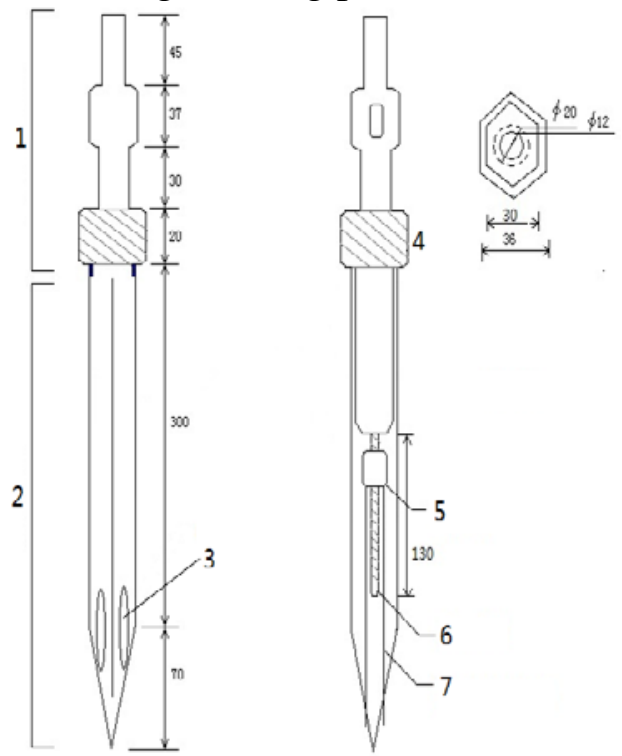

Fig.5 the mechanical design of the multi-needle grounding pole

Note: 1-- Connection to the power plant, 2--the portion inserted into soil, 3--The pinhole 4--Connecting nut, 5--Thimble nut, 6--Swivel nut, 7--Steel needle.

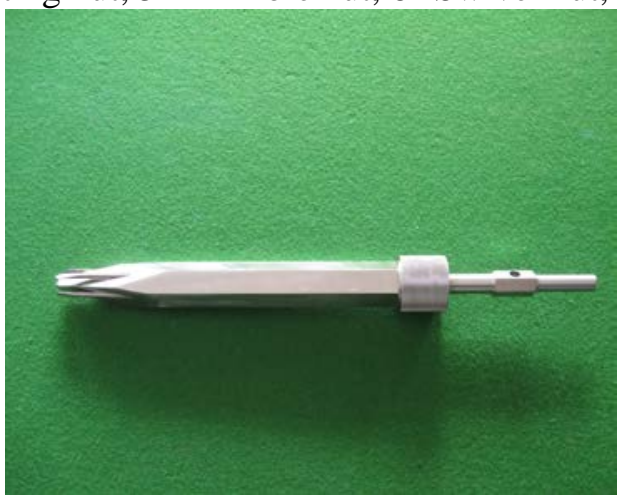

Fig.6 Picture of the multi-needle grounding pole

Choose $\mathrm{A}, \mathrm{B}, \mathrm{C}$ three tests spots again. We respectively using regular grounding pole, the inflatable type grounding pole and the multi-needle grounding pole on the test of grounding resistance, the measured data in the following table.

Table 2 The comparison table of grounding resistance test data

\begin{tabular}{|c|c|c|c|c|c|c|}
\hline & $\begin{array}{l}\text { regular } \\
\text { grounding pole }\end{array}$ & $\begin{array}{l}\text { Testing } \\
\text { time }\end{array}$ & $\begin{array}{l}\text { the inflatable type } \\
\text { grounding pole }\end{array}$ & $\begin{array}{l}\text { Testing } \\
\text { time }\end{array}$ & $\begin{array}{l}\text { the multi-needle } \\
\text { grounding pole }\end{array}$ & Testing time \\
\hline A zone & $6.6 \Omega$ & \multirow{3}{*}{$7 \mathrm{~min}$} & $7.2 \Omega$ & \multirow{3}{*}{$3 \mathrm{~min}$} & $3.7 \Omega$ & \multirow{3}{*}{$3 \mathrm{~min}$} \\
\hline B zone & $5.9 \Omega$ & & $6.1 \Omega$ & & $3.4 \Omega$ & \\
\hline C zone & $6.1 \Omega$ & & $6.6 \Omega$ & & $3.6 \Omega$ & \\
\hline
\end{tabular}

From the data in the table, the multi-needle grounding pole solves the shortcoming of the inflatable type grounding pole. When we use the multi-needle grounding pole to insert the soil, although we also use the application of the electric device, by the gearing effect of swivel nut, there 
are four steel needles whose diameter is $5 \mathrm{~mm}$,length is $130 \mathrm{~mm}$ on the top of grounding pole. Those steel needles contact with the soil closely. Make the result of tests are accurate, and it is very close to the grounding resistance data we got in $\mathrm{A}, \mathrm{B}, \mathrm{C}$ three test zones in the summer. It conforms to the regulations.

\section{The situation of application on spot}

From the date we start to research the multi-needle grounding pole, it can reach the actual requirement we need so far. The process of operation is simple, and it is doughtily application on spot. It can be applied to the rock, the frozen ground and the other hard permafrost soil. Using it, we can make the ground resistance testing wouldn't be affected by the seasonal environment influence. Make the test will not be affected by seasonal environment, to ensure the accuracy of results. Compared with the previous test, the multi-needle grounding pole is more effective.

\section{Summary}

Although the design and production of the multi-needle grounding pole is very complex, its application is convenient. It solves some practical problems. Therefore, it can be applied to the field work. Through the practical work, the method that we have used on the multi-needle grounding pole is correct and clear.

The multi-needle grounding pole has solved the problems which is not easy to insert into the rock, the frozen ground and the other hard permafrost soil. the multi-needle grounding pole has been widely used in Fushun. With good results, it has a wide range of promotional value and application prospects.

\section{References}

[1] Zhou Zecun. High Voltage Technology. Chinese Hydropower Press, 1996.12

[2] Chen Huagang. Preventability Testing Method and Diagnose Technique of Power Equipment. Beijing Technology Press, 2001

[3] Chen Jiabin. Grounding technology and grounding device. China Power Press, 2010

[4] Wang Changyu. Grounding Safety Technology. China Power Press, 2008

[5] Ping Shaoxun, Zhou Yufang. Operation Analysis And Grounding Mode In Electric System. China Power Press, 2010 